\title{
REDUCCIÓN DEL TIEMPO DE TRABAJO Y DESEMPLEO: UN ESCENARIO EUROPEO
}

\author{
Michel Husson ${ }^{1}$
}

DOI: $10.1387 /$ lan-harremanak.16570

\section{ABSTRACT}

En este artículo se señalan las condiciones que debe reunir la Reducción del Tiempo de Trabajo (RTT) para la creación de empleo, mediante un escenario a escala europeo y en base a un ejercicio realizado sobre Francia. Se expone las diferentes modalidades de reducción de la duración de trabajo: una, excluyente, basada en la coexistencia de jornadas a tiempo completo con jornadas a tiempo parcial y el desempleo; otra igualitaria, que reparte el total de las horas trabajadas entre todas las personas candidatas a un empleo. Se presenta un cálculo, sumario pero muy aclaratorio, sobre la "duración del trabajo de pleno empleo» que permitiría reducir a cero la tasa de paro. Asimismo se indica que la diferencia relativa entre la duración de trabajo de pleno empleo y la duración efectiva es igual a la tasa de paro. Se señalan las cuestiones politicas que van ligadas a una politica de reducción de la duración de trabajo y los aspectos de su financiación. Se concluye con los principios que debe reunir una "buena RTT». El artículo finaliza con tres breves anexos que presentan con matemáticas sencillas varios aspectos de la RTT.

Palabras clave: reducción tiempo de trabajo, trabajo, reparto, salario, empleo.

\footnotetext{
1 «Réduction du temps de travail et chômage : un scénario européen», nota hussonet n. ${ }^{\circ}$ 98, 26 de abril de 2006, http://goo.gl/4uycDH. Traducido por Mikel de la Fuente Lavín.
} 
This article describes the conditions that must include the working time reduction (WTR) in order to create employment. We limit at the European level and take as a reference a case developed for France. Our proposal includes two different modalities of reducing the duration of work: one in which full-time work, part-time work and unemployment coexist and another in which the total hours worked is distributed among all job applicants. The article exposes a concise but very explanatory calculation on the "working hours of full employment", which would reduce to zero the unemployment rate. We also note that the relative difference between the working hours of full employment and the effective hours is equal to the unemployment rate. Additionally we also identified the political and financial issues linked with a working time reduction policy. The article concludes with the principles that must include a "good WTR". As a complement, three short appendixes that present various aspects of WTR through easy mathematics have been incorporate.

Keywords: working time reduction (WTR), job sharing, wages, employment.

Artikulu honetan Lanaldiaren Murrizketak (LM) bete beharreko baldintzak azaltzen dira enplegua sortu ahal izateko, Europa mailako jokalekuan eta Frantziako kasuan oinarritu den ariketa batez. LM-ren bi aukera aurkezten dira: lehena, baztertzailea, lanaldi osoko enpleguarekin batera lanaldi partziala eta langabezia onartzen dituena; bigarrena, berdintasunezkoa, lan egindako ordu guztiak lan egin nahi duten pertsona guztien artean banatzen dituena. Kalkulu bide bat aurkezten da, laburra baina oso adierazgarria, enplegu beteko lanaldiari buruzkoa, langabezia-tasa zerora eramateko balioko lukeena. Artikuluan erakusten da ere enplegu beteko lanaldiaren eta benetako lanaldiaren arteko diferentzia erlatiboa langabeziatasaren berdina dela. LM baten finantziazioari eta kontu politiko batzuei buruzko zenbait iradokizun eskaintzen dira. Bukatzeko, LM "egoki» batek bete beharko lituzkeen printzipioen zerrenda egiten da. Amaieran, hiru eranskin laburretan, LMri buruzko hainbat gai matematika errazen bidez azaltzen dira.

Hitz gakoak: lanaldiaren murrizketa, lana, banaketa, soldata, enplegua. 
En el momento en el que la idea misma de reducción del tiempo de trabajo (RTT) es condenada como una herejía por los economistas neoliberales ${ }^{2}$, no es inútil mostrar en qué condiciones la RTT puede hacer retroceder al desempleo. Ilustramos esta demostración con ayuda de un escenario construido a escala europea, en base al modelo de un ejercicio realizado sobre Francia ${ }^{3}$.

\section{Y sin embargo se reduce}

Imaginemos una sociedad cuya población activa es de 100 personas: 60 de entre ellas trabajan a tiempo completo 40 horas por semana, otras 20 trabajan a tiempo parcial 20 horas por semana; y los 20 restantes están en desempleo y así pues a cero horas de trabajo. El número total de horas trabajadas es de $2.800 \mathrm{y}$ la duración media del trabajo (calculada sobre la base de las personas que tienen un empleo) es de 35 horas.

Consideremos ahora otra sociedad, que cuenta con la misma población activa y el mismo número de horas trabajadas. Pero estas están esta vez repartidas sobre el conjunto de la población activa. La duración del trabajo es entonces de 28 horas. Suponiendo que no existe el trabajo a tiempo parcial se observa que todos los empleos son de 28 horas.

Esta pequeña fábula permite ilustrar una realidad esencial: la cuestión no es saber si la duración del trabajo debe bajar o no sino cuales las modalidades de esta reducción, sea por la exclusión, sea por la igualdad. En Francia, el proyecto de ley «trabajo» está concebido para «descomponer» todavía un poco más las 35 horas e incluso vaciar de todo contenido la noción misma de duración legal del trabajo. Ha escogido así claramente la vía de la fragmentación social.

Por esta razón las comparaciones internacionales, muy a menudo manejadas para probar las virtudes de tal o cual «modelo» deber ser cuidadosamente desmenuzadas. En realidad, todos los países puestos como ejemplo porque han re-

\footnotetext{
2 Michel Husson, «Flexibilité du travail, arnaque néolibéral» (II), A l'encontre, 14 de marzo de 2016, http://goo.gl/jUUm7a

${ }^{3}$ Michel Husson, "Réduction du temps de travail et chômage: trois scenarios», A l'encontre, 4 de abril de 2016, http://goo.gl/6uh2Mn
} 
ducido su tasa de paro, sólo lo han realizado extendiendo el campo de la precariedad y del tiempo parcial.

\section{Pequeña aritmética de la RTT}

Nuestro ejemplo hipotético puede ser desarrollado sobre el ejemplo europeo. Se examinarán a continuación las objeciones habitualmente dirigidas a estas «reglas de tres» por los neoliberales que las encuentran simplistas. En Europa ${ }^{4}$, la producción de bienes y servicios ha necesitado en 2014284 mil millones de horas de trabajo (de las cuales el $80 \%$ aproximadamente de trabajo asalariado). La duración media de trabajo se calcula relacionando ese "volumen de trabajo» con el número de empleos. Siempre en 2014, el número de personas que disponían de un empleo era de 181 millones (de los que 154 millones eran asalariados). La duración anual del trabajo era pues de media de 1.575 horas y de 1.470 para los asalariados. Estas cifras son evidentemente globales y mezclan los empleos a tiempo pleno y a tiempo parcial.

Pero también se puede relacionar ese volumen de trabajo con la población activa, es decir con el conjunto de las personas en empleo o en desempleo, que se elevaba a 202 millones de personas (181 millones en empleo y 21 millones en paro). A partir de estos datos oficiales se puede también calcular la «duración del trabajo de pleno empleo» que sería pues de 1.409 horas por año.

Repartiendo de esta forma el volumen de trabajo entre todos los candidatos al empleo, se reduciría a cero la tasa de paro. La diferencia relativa entre la duración de trabajo de pleno empleo y la duración efectiva es en efecto igual a la tasa de paro, en este caso del $10,5 \%=(1.575-1.409) / 1.575$ (ver gráfico $1 \mathrm{y}$ anexo 1).

Para suprimir el paro en Europa, ese cálculo muestra que sería necesario reducir la duración del trabajo en aproximadamente el 10\%. Como la duración media equivale a aproximadamente 39 horas sería pues necesario pasar a las 35 horas.

\footnotetext{
414 países: Austria, Bélgica, Dinamarca, España, Finlandia, Francia, Grecia, Irlanda, Italia, Luxemburgo, Holanda, Portugal, Reino Unido, Suecia.
} 
Gráfico 1

Duración efectiva del trabajo y duración del trabajo de pleno empleo

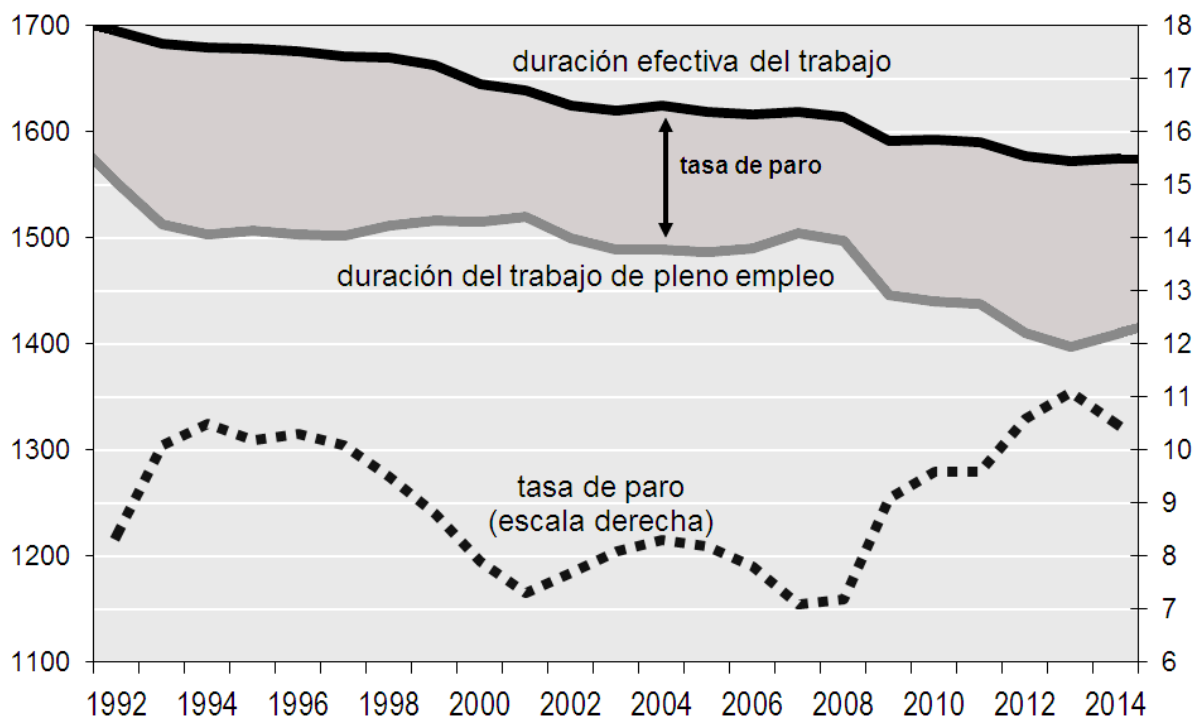

\section{Un escenario alternativo}

Este escenario se basa sobre una hipótesis de reducción más rápida de la duración del trabajo que la observada en Europa: en lugar de caer de media de $0,33 \%$ por año, habría bajado el $0,6 \%$ por año, con las mismas inflexiones coyunturales que la duración observada. En 2014, la duración del trabajo sería entonces el 5,7\% inferior a su nivel observado (ver gráfico 2.A). Esta reducción simulada solo franqueó pues una parte del camino hacia la duración del trabajo de pleno empleo. Ella permite sin embargo reducir de forma significativa la tasa de paro que habría sido inferior al 6\% desde el inicio de los años 2000 (ver gráfico 2.B).

Este escenario no es neutro en lo que concierne al reparto de los ingresos. Si la reducción suplementaria de la duración del trabajo no es "compensada» por una baja del salario, la parte de los salarios debe lógicamente aumentar. Pero este aumento no hace más que recuperar una parte del retroceso registrado desde el inicio de los años 1980 (ver gráfico 2.C). 


\section{Gráfico 2}

\section{Reducción de la duración del trabajo: un escenario europeo}

A. Duración del trabajo

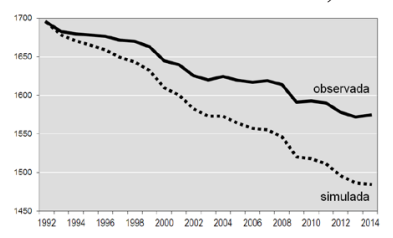

B. Tasa de paro

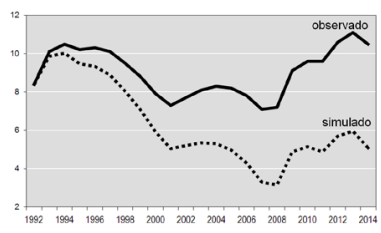

C. Parte de los salarios

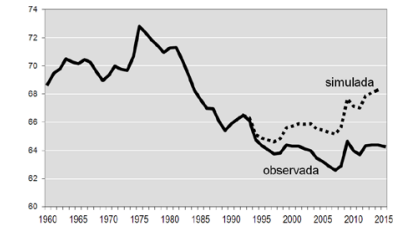

\section{El cuestionamiento de las hipótesis}

El ejercicio que acaba de presentarse se basa en la hipótesis implícita según la cual están dadas tres magnitudes: el volumen de trabajo, la población activa y la productividad del trabajo. Ellas suscitan a priori numerosas objeciones que han sido especialmente planteadas por los economistas neoliberales opuestos por principio a una política de reducción del tiempo de trabajo.

Considerar como dado el volumen de trabajo sería un razonamiento «maltusiano». Pero, en la práctica, solo aumentó el 6,2\% entre 1992, o sea el 0,3\% anual. En Francia ha progresado ligeramente entre 1997 y 2002, contrariamente a las predicciones de los detractores de la RTT.

Esta constatación puede interpretarse observando que la tasa de crecimiento del volumen de trabajo es la diferencia entre la tasa de crecimiento del PIB y la de la productividad horaria del trabajo (ver anexo 2). Sin embargo, se observa que, sobre un amplio período, el PIB y la productividad horaria tienden a aumentar a la misma tasa, de tal forma que el volumen de trabajo tiende a permanecer constante, o a aumentar muy moderadamente.

La segunda objeción es aceptable: la población inactiva no está inerte. Puede, por ejemplo, aumentar cuando el mercado de trabajo va mejor: las personas van en efecto a intentar encontrar un empleo, mientras que renuncian cuando aumenta la tasa de paro. Es lo que los economistas llaman «efecto de flexión» (ver anexo 3). Sería pues necesario, para proceder rigurosamente, modificar el modelo. La toma en cuenta de este "efecto de flexión" reduciría a corto plazo el impacto de la reducción del tiempo de trabajo, pero este efecto se disiparía a medio plazo.

La tercera objeción (la producción del trabajo está dada) se combina con la primera, y se apoya en dos razonamientos. La reducción de la duración del trabajo sin pérdida de salario haría aumentar el «coste del trabajo» y tendría, vía esta pérdida de competitividad, un impacto negativo sobre el nivel de activi- 
dad y, así, sobre el empleo. O bien, las ganancias de productividad reducirían el efecto sobre el empleo.

\section{Una opción de sociedad, una cuestión política}

Estas dos últimas objeciones permiten señalar que el ejercicio presentado no proviene de la economía "pura» sino que plantea cuestiones políticas. El argumento de la competitividad supone implícitamente que no se toca a la parte de los beneficios que va a dividendos. Sin embargo, esta es precisamente la contrapartida de una reducción insuficiente de la duración del trabajo y, con ello, del paro. No es coherente querer reducir este sin cuestionar la punción accionarial. La creación de empleos por reducción del tiempo de trabajo, y sin pérdida de salario, aumenta evidentemente la masa salarial; pero ella puede ser perfectamente compensada por una reducción de los dividendos distribuidos a los accionistas ${ }^{5}$.

Por otra parte, no hay que olvidar que la creación de empleos por reducción del tiempo de trabajo se «autofinancia» parcialmente. Incluso sin hablar de sus efectos sociales e individuales sobre el bienestar, el desempleo no es gratuito. A las prestaciones de desempleo hay que ańadir los efectos indirectos, especialmente en materia de salud pública. Reabsorber el desempleo reduce pues su coste en una proporción que, en el caso francés, va de un tercio a la mitad de un salario. Sería pues posible reducir las cotizaciones a medida que los empleos creados reduzcan las sumas dedicadas a la indemnización del desempleo, y el aumento de la parte de los salarios sería reducida otro tanto.

En cuanto al argumento de a productividad, es exacto: si la reducción de la duración del trabajo se compensa con una intensificación del trabajo, entonces es cierto que se reduce el efecto sobre el empleo. Ello es lo que ha sucedido durante el paso a las 35 horas en Francia: una parte del efecto de la reducción del tiempo de trabajo ha sido anulado por diversas formas de intensificación del trabajo. De esta importante experiencia se pueden extraer en conclusión varias lecciones de aplicación general.

Los principios de una buena RTT

— Mantenimiento del salario mensual: la RTT no debe ser un reparto del trabajo a masa salarial constante.

— La RTT debe ponerse en práctica «con contrataciones compensatorias» proporcionales a fin de evitar toda intensificación del trabajo: 10\% de

5 Para una demostración más detallada, ver Michel Husson, «Soixante ans d'emploi», 2009, http://goo.gl/x99OhY; ou Michel Husson, «Unemployment, working time and financialisation: the French case», Cambridge Journal of Economics, vol.39 n. ${ }^{3}$, 2015, https://goo.gl/zB27Io 
reducción del tiempo de trabajo $=10 \%$ de empleos de más (¡e incluso el $11,1 \%$ por las leyes de la aritmética!).

— La RTT debe ser la ocasión de una reabsorción del trabajo a tiempo parcial sufrido.

— La RTT debe acompañarse de formas de mutualización de los empleos en las pequeñas empresas.

Pero la condición de éxito más importante es el control de los asalariados sobre la puesta en práctica de la medida. Este control debe consistir en dos puntos esenciales: el primero es la realidad de las creaciones de empleos, el segundo las modalidades concretas de la RTT. Son los trabajadores quienes deben decidir colectivamente sobre la mejor forma de combinar las formas posibles de reducción del tiempo de trabajo, de forma que se tomen en cuenta las aspiraciones diferenciadas de los asalariados: reducción diaria (tantas horas por día), semanal (por ejemplo 4 días), anual (jornadas RTT) o plurianual (año sabático). Una forma de ese control podría ser la siguiente: en la medida en que los gastos del desempleo disminuirían con las creaciones de empleos, podrían ser acordadas reducciones de las cotizaciones sociales. Pero ellas serían sometidas a la validación por los asalariados respecto a cuatro criterios: la realidad de las creaciones de empleos, su carácter decente, el mantenimiento de los salarios y una política de reducción de los abonos de dividendos.

\section{Anexos}

\section{Duración del trabajo de pleno empleo y tasa de desempleo}

Sea $\mathrm{N}$ el empleo, VOL el número total de horas de trabajo, DES el desempleo y POPAC la población activa. La duración media del trabajo DAT se calcula según: $\mathrm{DAT}=\mathrm{VOL} / \mathrm{N}$

La duración del trabajo de pleno empleo DATPE se calcula según: DATPE $=$ VOL $/$ POPAC

Pero si se tiene en cuenta el hecho de que POPAC $=\mathrm{N}+\mathrm{DES}$, la ratio DATPE/DAT puede escribirse:

DATPE $/$ DAT $=\mathrm{N} / \mathrm{POPAC}=1-\mathrm{TDES}$ con TDES $($ tasa de desempleo $)=\mathrm{DES} / \mathrm{POPAC}$

Se alcanza pues:

$$
\text { TDES }=(\text { DAT }- \text { DATPE }) / D A T
$$




\section{PIB y productividad horaria}

La productividad horaria (PRODH) se calcula relacionando el PIB con el número de horas trabajadas (VOL). Se tiene pues PRODH $=$ PIB/VOL. Entonces es fácil deducir que: $\mathrm{VOL}=\mathrm{PIB} / \mathrm{PRODH}$, o anotando como tx a la tasa de crecimiento:

$$
\mathrm{txVOL}=\mathrm{txPIB}-\mathrm{txPRODH} .
$$

\section{El efecto de flexión}

La población activa (POPAC) es por definición la suma del empleo $(\mathrm{N})$ y del desempleo $(\mathrm{DES})$. En variaciones $(\Delta)$, se tiene entonces: $\triangle \mathrm{DES}=\triangle \mathrm{POPAC}-\Delta \mathrm{N}$. Pero si el aumento del empleo $(\Delta \mathrm{N}=10)$ induce un aumento de la población activa (por ejemplo $\triangle \mathrm{POPAC}=5$ ), entonces la caída del desempleo $(\triangle \mathrm{DES})$ es de 5 , es decir inferior a las creaciones de empleos. 